\section{Integrated mRNA and Small RNA Sequencing for Analyzing Leaf Spot Pathogen Didymella segeticola and Its Host, Tea (Camellia sinensis), During Infection}

\author{
Rui Yang, ${ }^{1,2}$ Silong Jiang, ${ }^{1,2}$ Dongxue Li, ${ }^{1}$ Qiaoxiu Yin, ${ }^{1}$ Xian Wu, ${ }^{1}$ Yong Wang, ${ }^{2}$ Delu Wang, ${ }^{3, \dagger}$ and \\ Zhuo Chen ${ }^{1, \dagger}$ \\ ${ }^{1}$ Key Laboratory of Green Pesticide and Agricultural Bioengineering, Ministry of Education, Guizhou \\ University, Guiyang, Guizhou 550025, China \\ ${ }^{2}$ Agricultural College, Guizhou University, Guiyang, Guizhou 550025, China \\ ${ }^{3}$ College of Forestry, Guizhou University, Guiyang, Guizhou 550025, China
}

\begin{abstract}
Leaf spot on tea plants (Camellia sinensis [L.] Kuntze), caused by the fungus Didymella segeticola (Q. Chen) Q. Chen, Crous \& L. Cai (syn. Phoma segeticola), negatively affects the productivity and quality of tea leaves in Guizhou Province, China. Although the genome sequence of $D$. segeticola has been published, no data on the transcriptome or microRNAs (miRNAs) of the pathogen or host during infection are available. Here, we report on the high-quality transcriptome and miRNA sequences of both $D$. segeticola and tea during infection, using the Illumina HiSeq 4000 or HiSeq 2500 platforms. Comprehensive expression profiling of the fungal pathogen and its host will provide a resource for future research into trait-specific genes of the pathogen and the host as well as on hostpathogen interactions and on disease resistance mechanisms.
\end{abstract}

\section{Transcriptome and microRNA Sequence Announcement}

The Didymellaceae family consists of 19 monophyletic generic clades, each representing an individual genus, which include many phytopathogens, causing diseases, decreased production, and quality in a number of crops (Chen et al. 2017). Didymella segeticola, in the clade of Didymella, can cause leaf spot on Tibetan thistle (Chen et al. 2015) and on the tea plant (C. sinensis) (Ren et al. 2019; Zhao et al. 2018). The whole genome of $D$. segeticola was recently sequenced and annotated by our research group (BioProject PRJNA516041) (Ren et al. 2019).

A single conidium of $D$. segeticola GZSQ-4, isolated from leaf spot-infected tea leaves in Ghuizou Province, was incubated on potato dextrose agar (PDA) at $28^{\circ} \mathrm{C}$ in the dark for 10 days. Five-year-old potted tea plants of $C$. sinensis cv. Fudingdabaicha grown in the greenhouse were maintained at $25^{\circ} \mathrm{C}$ during the day and $20^{\circ} \mathrm{C}$ at night, with cycles of $14-\mathrm{h}$ light and 10-h dark, with a relative humidity of 70 to $80 \%$. Healthy twigs were selected at the one-bud and fifth-leaf stages for the inoculation experiment ( $\mathrm{Li}$ et al. 2019). Tea leaves were punctured by a sterile needle, were then inoculated with either a 20 - to $30-\mu$ l suspension of D. segeticola GZSQ-4 conidia ( $10^{6}$ conidia per milliliter of water per inoculation site) as the

${ }^{\dagger}$ Corresponding authors: D. Wang; fc.dlwang@gzu.edu.cn and Z. Chen; gychenzhuo@aliyun.com

"The $e$-Xtra logo stands for "electronic extra" and indicates supplementary figure and table files are published online.

The author(s) declare no conflict of interest.

Accepted for publication 30 September 2020.

$e-\mathrm{Xtra}^{*}$

Funding

The project was supported by National Key Research Development Program of China (2017YFD0200308) and Post-subsidy project of National Key Research Development Program of China (2018-5262), the National Natural Science Foundation of China (numbers 31860515 and 21977023), Program of Introducing Talents to Chinese Universities (111 Program, D20023) and the China Agriculture Research System (CARS-23-D09).

\section{Keywords}

Didymella segeticola, fungus-plant interactions, microRNA, leaf spot, tea, transcriptome 
Table 1. Statistics of transcriptome and microRNA (miRNA) sequences of Didymella segeticola and tea during infection

\begin{tabular}{|c|c|c|c|c|c|c|}
\hline \multirow[b]{3}{*}{ Sequence/kind of data } & \multicolumn{6}{|c|}{ Sample source ${ }^{a}$} \\
\hline & \multicolumn{4}{|c|}{ Mycelia } & \multicolumn{2}{|c|}{ Tea leaf } \\
\hline & Treatment 1 & Treatment 2 & Treatment 3 & Treatment 4 & Treatment 3 & Treatment 4 \\
\hline \multicolumn{7}{|l|}{ Transcriptome } \\
\hline Raw reads & $43,343,284$ & $46,704,947$ & $46,410,408$ & $47,926,218$ & $46,410,408$ & $47,926,218$ \\
\hline Valid reads & $39,272,608$ & $40,684,663$ & $45,522,197$ & $47,071,604$ & $45,522,197$ & $47,071,604$ \\
\hline Valid bases & $5.89 \mathrm{G}$ & $6.10 \mathrm{G}$ & $6.83 \mathrm{G}$ & $7.06 \mathrm{G}$ & $6.83 \mathrm{G}$ & $7.06 \mathrm{G}$ \\
\hline Transcripts from transcriptome & 10,894 & 10,894 & NA & 10,894 & 33,932 & 33,932 \\
\hline Unigenes from transcriptome & 10,894 & 10,894 & NA & 10,894 & 33,932 & 33,932 \\
\hline \multicolumn{7}{|l|}{ miRNA } \\
\hline Raw reads & - & - & $16,673,700$ & $13,651,746$ & $16,673,700$ & $13,651,746$ \\
\hline Valid reads & - & - & $13,335,514$ & $10,804,656$ & $13,335,514$ & $10,804,656$ \\
\hline
\end{tabular}

infected treatment or 20 to $30 \mu$ l of sterile water as the control treatment. Total RNA containing $C$. sinensis or $D$. segeticola mycelium from the infected treatment and the control treatment was isolated from inoculated tea leaves. After inoculation for $48 \mathrm{~h}$, a lesion had formed rapidly on each leaf inoculated with conidia but not on those inoculated with water. A single conidium culture of $D$. segeticola GZSQ-4 on PDA or PDA containing matcha (green tea leaf powder, $0.2 \mathrm{~g}$ per liter) at $28^{\circ} \mathrm{C}$ for 10 days was used as sources of $D$. segeticola RNA. Each treatment was set to three biological replicates.

Total RNA of tea leaf (48 $\mathrm{h}$ after inoculation with conidia or water) or Didymella mycelium(cultured in the presence or absence of matcha) were extracted using TRIzol reagent (Invitrogen, Carlsbad, CA, U.S.A.), following procedures of the manufacturer. The cDNA libraries with three biological replicates were prepared using the mRNA-Seq sample preparation kit (Illumina, San Diego, CA, U.S.A.) and were sequenced on an Illumina HiSeq 4000 at Lc-Bio Technologies Co., Ltd. (Hangzhou, China), following the manufacturerrecommended protocol. Cutadapt (Martin 2011) and in-house Perl script were used to remove the low-quality reads, including adapters, low-quality bases, and undetermined bases. Then, the sequence quality was verified using FastQC, including the Q20 and Q30 indexes, and the GC content of the cleaned data. All downstream analyses were based on clean data of high quality. De novo assembly of the transcriptome was performed with Trinity 2.4.0 (Grabherr et al. 2011). Unigenes were assembled using StringTie software (Pertea et al. 2015) and differential expression analysis was conducted using edgeR software (threshold value of significant difference with $\log _{2}^{\text {foldchange }} \geq 1, P<0.05$ ). All assembled unigenes were annotated using Gene Ontology (GO), Kyoto Encyclopedia of Genes and Genomes (KEGG), Protein Families (Pfam), Swiss-Prot, eggnog, and the National Center for Biotechnology Information nonredundant protein databases, using DIAMOND software (Buchfink et al. 2015).

The number of annotated pathogen and host unigenes was 10,894 and 33,932, respectively (Table 1). The number of differentially expressed genes in the tea plant during infection, compared with control treatment, was 678 upregulated and 1,222 downregulated genes. In the GO aspect of biological process, the top three enriched GO terms were the oxidation-reduction process, protein phosphorylation, and carbohydrate metabolic process, with the number of ' $S$ ' genes (genes with significant differential expression as annotated in special GO) being 102, 46, and 21, respectively. The top three enriched GO terms at the level of cellular component were integral component of membrane, membrane, and nucleus, with the number of $S$ genes being 138,46 , and 27 , respectively. The top three enriched $G O$ terms at the level of molecular function were integral component of ATP binding, oxidoreductase activity, and metal ion binding, with the number of $S$ genes being 82, 37, and 34, respectively. The pathways of plant-pathogen interaction, starch and sucrose metabolism, phenylpropanoid biosynthesis, plant hormone signal transduction, and flavonoid biosynthesis was significantly enriched in the inoculated leaves relative to the control leaves, with the numbers of genes upregulated in each of these five terms being $81,41,31,28$, and 20 , respectively. 
The library of small RNAs was prepared using TruSeq Small RNA sample prep kits (Illumina), and sequenced by Illumina HiSeq 2500 (Illumina), with single-end for sequencing and a sequencing read length of $1 \times 50 \mathrm{bp}$. Raw reads were analyzed using ACGT101-miR (LC Sciences, Houston, TX, U.S.A.) to remove adapter dimers and low-complexity sequences, were filtered by aligning the databases of mRNA, RFam (containing rRNA, transfer RNA, small nuclear RNA, and small nucleolar RNA), and Repbase. The reads with lengths of 18 to $25 \mathrm{nt}$ (microRNA [miRNA]-like) were retained, and unique sequences with lengths in the range 18 to $25 \mathrm{nt}$ were mapped to species-specific precursors in miRBase 22.0 by BLAST search, to identify known miRNAs and novel 3p- and 5p-derived miRNAs. The rest of the sequences were aligned through the databases of mRNA, RFam, and Repbase, and were filtered to form valid data. The significance of differential expression of miRNAs was analyzed by Student's $t$ test. Target genes of miRNAs with differential expression were predicted using TargetFinder (Dai and Zhao 2011), and their enrichment analysis was conducted using GO, SwissProt, and KEGG.

MiRNAs were identified and predicted and were then divided into gp1, gp2a, gp2b, gp3, and gp4. Up-/down-regulation of differentially expressed miRNAs were 17/15, 88/92, 209/215 for infected treatment versus control treatment at the level of $P$ value being $0.01,0.05$, and 0.1 , respectively. The target genes of the miRNAs were predicted; miRNAs PC-3p-9306445_2, nta-MIR6149a-p3_2ss13GA18CG, mdm-MIR535a-p3_2ss13GA18CG, and cst-MIR11334p3_2ss13GA18CG targeted the most genes, with the number of target genes for each miRNA being $31,21,16$, and 11, respectively. The predicted target genes of the miRNAs were annotated using $\mathrm{GO}$, with the number of $\mathrm{S}$ genes being significantly enriched with respect to protein phosphorylation or the oxidation-reduction process at the level of biological process being 6 and 4, respectively. At the level of the cellular component aspect, integral component of membrane, membrane, nucleus, and plasma membrane were significantly enriched, with the number of S genes being 24, 9, 8, and 8, respectively. Significant enrichment of the predicted target genes of miRNAs, determined by analysis using the KEGG database, revealed significant enrichment with respect to the pathways of plant hormone signal transduction, plant-pathogen interaction, and toxoplasmosis, with the number of $S$ genes being 14, 11 and 10, respectively. Correlation analysis of transcriptome and miRNA data for tea leaf was shown as the category of regulation relation for differential correlation data, using Integrated Analysis For Three Types (version 2.4), and the methods were briefly described as follows: i) target genes of miRNA were predicted, ii) data of miRNA and mRNA were correlated, iii) transcriptome data and gene data were correlated, iv) data with significant difference and regulation relation was screened at the level of mRNA (false discovery rate $<0.05$, fold change $>2$ or $<0.5$ ) and miRNA ( $P$ value $<0.05$ ); and v) enrichment analysis of GO and KEGG was conducted using GO and KEGG databases. Eighteen genes in the transcriptome and miRNA data were validated by quantitative reverse-transcription PCR, from the samples of infected treatment and uninfected treatment at 2, 4, and 6 days after inoculation.

The sequences of the transcriptome and the miRNAs of $D$. segeticola will provide an important resource for researchers studying pathogenic mechanisms and disease resistance responses, specifically for the $D$. segeticola- $C$. sinensis interaction. The sequences of the transcriptome and miRNAs of $D$. segeticola have been deposited in GenBank under Sequence Read Archive accession numbers PRJNA648680 and PRJNA528172 for transcriptome data and PRJNA534364 for miRNA data. In addition, bioinformatics analysis of transcriptomes and miRNAs are presented in Supplementary Table S1 and Supplementary Figure S1. D. segeticola GZSQ-4 has been deposited in the China General Microbiological Culture Collection, with the preservation number of the fungus as CGMCC3.20152.

\section{Author-Recommended Internet Resources}

DIAMOND software: http://ab.inf.uni-tuebingen.de/software/diamond

eggnog: http://eggnog5.embl.de

FastQC: http://www.bioinformatics.babraham.ac.uk/projects/fastqc

Gene Ontology (GO) database: http://www.geneontology.org

Kyoto Encyclopedia of Genes and Genomes (KEGG): http://www.genome.jp/kegg

miRBase 22.0: ftp://mirbase.org/pub/mirbase/CURRENT

National Center for Biotechnology Information nonredundant protein database: http://www.ncbi.nlm.nih.gov

Pfam database: http://pfam.xfam.org

Repbase: http://www.girinst.org/repbase

RFam: http://rfam.janelia.org

StringTie software: https://ccb.jhu.edu/software/stringtie

Vol. 34, No. 1, $2021 / 129$ 


\section{Literature Cited}

Buchfink, B., Xie, C., and Huson, D. H. 2015. Fast and sensitive protein alignment using DIAMOND. Nat. Methods 12:59-60.

Chen, Q., Hou, L. W., Duan, W. J., Crous, P. W., and Cai, L. 2017. Didymellaceae revisited. Stud. Mycol. 87:105-159.

Chen, Q., Zhang, K., Zhang, G., and Cai, L. 2015. A polyphasic approach to characterise two novel species of Phoma (Didymellaceae) from China. Phytotaxa 197:267-281.

Dai, X., and Zhao, P. X. 2011. psRNATarget: A plant small RNA target analysis server. Nucleic Acids Res. 39 (suppl_2):W155-W159.

Grabherr, M. G., Haas, B. J., Yassour, M., Levin, J. Z., Thompson, D. A., Amit, I., Adiconis, X., Fan, L., Raychowdhury, R., Zeng, Q., Chen, Z., Mauceli, E., Hacohen, N., Gnirke, A., Rhind, N., di Palma, F., Birren, B. W., Nusbaum, C., Lindblad-Toh, K., Friedman, N., and Regev, A. 2011. Full-length transcriptome assembly from RNASeq data without a reference genome. Nat. Biotechnol. 29:644-652.
Li, D. X., Bao, X. T., Ren, Y. F., Wang, Y., Song, B. A., and Chen, Z. 2019. First report of Lasiodiplodia theobromae causing leaf spot on tea plant in Guizhou Province of China. Plant Dis. 103:374.

Martin, M. 2011. Cutadapt removes adapter sequences from high-throughput sequencing reads. EMBnet. J. 17:10-12.

Pertea, M., Pertea, G. M., Antonescu, C. M., Chang, T. C., Mendell, J. T., and Salzberg, S. L. 2015. StringTie enables improved reconstruction of a transcriptome from RNA-seq reads. Nat. Biotechnol. 33:290-295.

Ren, Y., Li, D., Zhao, X., Wang, Y., Bao, X., Wang, X., Wu, X., Wang, D., Song, B., and Chen, Z. 2019. Whole genome sequences of the tea leaf spot pathogen Didymella segeticola. Phytopathology 109:1676-1678.

Zhao, X., Wang, Y., Li, D., Ren, Y., and Chen, Z. 2018. Morphological characterization and phylogenetic analysis of the pathogen Phoma segeticola var. camelliae causing a new tea disease. Acta Phytopathol. Sin. 48: 556-559. 